\title{
Inhibiting miR-1 attenuates pulmonary arterial hypertension in rats
}

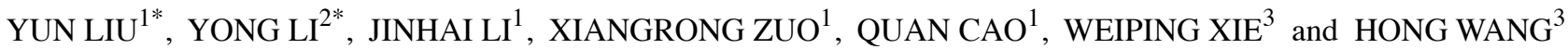 \\ Departments of ${ }^{1}$ Intensive Care Medicine,${ }^{2}$ Cardiology and ${ }^{3}$ Respiratory and Critical Care Medicine, \\ The First Affiliated Hospital of Nanjing Medical University, Nanjing, Jiangsu 210029, P.R. China
}

Received April 11, 2020; Accepted January 27, 2021

DOI: $10.3892 / \mathrm{mmr} .2021 .11922$

\begin{abstract}
MicroRNAs (miRs) are reported to serve key roles in pulmonary arterial hypertension (PAH). miR-1 has been found in cardiovascular diseases. The present study aimed to determine whether the knockdown of miR-1 could inhibit right ventricle (RV) remodeling and thereby control $\mathrm{PAH}$ in model rats. PAH model rats were established by exposing rats to hypoxia, while cardiac fibroblasts (CFs) obtained from PAH model rats were treated with hypoxia to establish an in vitro model, and RV remodeling was evaluated by Masson staining and the levels of collagen I, collagen III, $\alpha$-smooth muscle actin ( $\alpha$-SMA) and connective tissue growth factor (CTGF) evaluated by western blotting or reverse transcription-quantitative PCR. The results revealed that the expression levels of miR-1 were upregulated in the RV of PAH model rats induced with hypoxia and in the CFs treated with hypoxia. The mean pulmonary arterial pressure, RV systolic pressure, $\mathrm{RV} /($ left ventricle + interventricular septum) and RV/tibia length were increased in PAH rats; however, the increases in all parameters were subsequently reversed by transfection with a miR-1 antagomiR in PAH model rats. The transfection with the miR-1 antagomiR inhibited the development of RV fibrosis and downregulated the mRNA expression levels of collagen I, collagen III, $\alpha$-SMA and CTGF in the RV tissue of PAH model rats. The upregulation of collagen I, collagen III, $\alpha$-SMA and CTGF expression levels in hypoxia-treated CFs was also subsequently reversed by miR-1 antagomiR transfection. The expression levels of collagen I, collagen III, $\alpha$-SMA and CTGF were also upregulated in the CFs obtained from PAH model
\end{abstract}

Correspondence to: Dr Hong Wang, Department of Respiratory and Critical Care Medicine, The First Affiliated Hospital of Nanjing Medical University, 300 Guangzhou Road, Nanjing, Jiangsu 210029, P.R. China

E-mail: hong_wangnj@163.com

${ }^{*}$ Contributed equally

Key words: microRNA-1, pulmonary hypertension, hypoxia, fibrosis, cardiac fibroblasts rats, and these increases were attenuated by miR-1 antagomiR transfection. The expression levels of phosphorylated (p)-PI3K and p-AKT were also upregulated in hypoxia-treated CFs, and these increases were also inhibited by transfection with miR-1 antagomiR. In conclusion, these results indicated that inhibiting miR-1 may attenuate RV hypertrophy and fibrosis in PAH model rats, a mechanism that may involve the PI3K/AKT signaling pathway.

\section{Introduction}

Pulmonary arterial hypertension (PAH) is a pathological condition that occurs in the cardiovascular system (1). In PAH, pulmonary vascular resistance and pulmonary artery pressure increase, ultimately resulting in right heart failure and even death $(2,3)$. Maladaptive processes, such as fibrosis, can damage or even collapse the function of the right ventricle (RV) (4).

MicroRNAs (miRNAs/miRs) are a group of small endogenous non-coding RNAs that can negatively regulate target gene expression post-transcriptionally, mainly through mRNA degradation or translational inhibition (5-8). Alterations in the expression levels of miRNAs have been associated with a number of pathological disease processes, such as cardiovascular diseases. For this reason, circulating miRNAs have been hypothesized to be potential biomarkers or therapeutic targets for several types of disease, such as miR-29 in atrial fibrillation and miR-133a in myocardial infarction $(9,10)$. In fact, several miRNAs, including miR-143, miR-124, miR-140-5p and miR-135a, have been reported to be dysregulated in PAH animal models or patients with PAH (11-14).

Previous studies have revealed that miR-1 was involved in the pathogenesis of left heart failure and left ventricle (LV) fibrosis $(15,16)$. Dysregulated miR-1 biogenesis was previously associated with heart failure in aged rats, especially aged hypertensive rats (17). In addition, the expression levels of miR-1 were upregulated in lungs from an experimental model of PAH and in the plasma from patients with $\mathrm{PAH}$, and miR-1 induced endothelial dysfunction, suggesting a pathophysiological role for miR-1 in PAH (18). In a previous study, the transfection with the miR1 antagomiR downregulated the expression levels of TGF- $\beta$ and collagen hyperplasia in myocardial infarction model mice (19). However, to the best of our knowledge, whether miR-1 may be involved in the regulation of $\mathrm{PAH}$ remains unknown. 
The PI3K/AKT signaling pathway was discovered to be involved in the regulation of cardiac fibrosis (20). A previous study revealed that miR-132 activated the PI3K/AKT signaling pathway by downregulating PTEN expression levels, thus inhibiting apoptosis and facilitating cardiomyocyte proliferation and cardiac fibrosis in dilated cardiomyopathy model rats (21). However, whether the PI3K/AKT signaling pathway may be involved in the regulatory effects of miR-1 on cardiac fibrosis in PAH remains unclear.

The present study aimed to determine whether the knockdown of miR-1 could counter PAH through attenuating $\mathrm{RV}$ fibrosis in PAH model rats, and whether the PI3K/AKT signaling pathway may be involved in the key roles of miR-1 in regulating fibrosis in CFs.

\section{Materials and methods}

Animal studies. Experiments were performed using 78 5-6 weeks-old male Sprague-Dawley (SD) rats (weight, 180-200 g; Beijing Vital River Laboratory Animal Technology Co., Ltd.). All procedures were approved by the Experimental Animal Care and Use Committee of Nanjing Medical University (Nanjing, China; approval no. 17041015), and were conducted in accordance with the Guide for the Care and Use of Laboratory Animals (National Institutes of Health publication no. 85-23, revised 1996) (22). The rats were kept in a temperature $\left(22 \pm 1^{\circ} \mathrm{C}\right)$ and humidity (40-60\%)-controlled room under a 12-h light/dark cycle with free access to standard chow and tap water. The experiments were performed at the Animal Core Facility of Nanjing Medical University.

Establishment of hypoxia rat model and grouping. The establishment of the hypoxic condition was performed as previously described (23). Briefly, SD rats were divided into 2 groups: i) Normoxia group $(n=8)$, in which rats received normoxia $\left(21 \% \mathrm{O}_{2}\right)$ for 4 weeks; and ii) hypoxia group $(\mathrm{n}=13)$, in which rats received hypoxia $\left(10 \% \mathrm{O}_{2}\right)(24,25)$ for 4 weeks. The expression levels of miR-1 were subsequently determined in the RV of rats in the two groups.

In another experiment, the 5-6 weeks-old rats were divided into the following groups: i) Normoxia + negative control (NC) antagomiR ( $\mathrm{n}=10)$; ii) normoxia + miR-1 antagomi $(\mathrm{n}=10)$; iii) hypoxia + NC antagomiR ( $n=15)$; and iv) hypoxia + miR-1 antagomiR groups $(n=15)$. Hypoxia and normoxia were administered as aforementioned. Simultaneously, rats were injected with miR-1 antagomiR (sequence 5'-UGGAAUGUAAAGAAG UGUGUAU-3'; Guangzhou RiboBio Co., Ltd.) or NC antagomiR (sequence 5'-CAGUACUUUUGUGUAGUACAA-3'; Guangzhou RiboBio Co., Ltd.) via the tail vein twice a week ( $40 \mathrm{mg} / \mathrm{kg} / \mathrm{time})$. After 4 weeks, RV function and fibrosis were determined.

Animal experiments. SD rats were anesthetized with $50 \mathrm{mg} / \mathrm{kg}$ pentobarbital (i.p.). Using a PowerLab data acquisition system (ADInstruments, Ltd.), a 1.4F conductance micromanometer catheter (Millar) was inserted via the RV, across the aortic valve and into the RV chamber to measure the right ventricular systolic pressure (RVSP) and the mean pulmonary arterial pressure (mPAP). Subsequently, the rats were sacrificed by cervical dislocation following anesthesia with $3.5 \%$ isoflurane induction and $2 \%$ isoflurane maintenance. The $\mathrm{RV}, \mathrm{LV}$ and interventricular septum (S) of the rats were separately dissected. The tibia length (TL) was measured and weighed to calculate the ratio of $\mathrm{RV}$ to $(\mathrm{LV}+\mathrm{S})$ and $\mathrm{RV} / \mathrm{TL}$, two key indicators for assessing RV hypertrophy.

Isolation and culture of cardiac fibroblasts (CFs). Rat CFs were isolated from 60 male and female SD rats (age, 1-3 days old; weight, 5-8 g; Beijing Vital River Laboratory Animal Technology Co., Ltd.), or male 9-10 weeks-old PAH (PCFs) or normoxia (NCFs) model rats (350-400 $\mathrm{g} ; \mathrm{n}=6$ for each group). The rats were kept in a temperature $\left(22 \pm 1^{\circ} \mathrm{C}\right)$ and humidity (40-60\%)-controlled room under a $12 \mathrm{~h}$ light-dark cycle with free access to standard chow and tap water. The rats were sacrificed by cervical dislocation following anesthesia with $3.5 \%$ isoflurane induction for $2 \mathrm{~min}$ and $2 \%$ isoflurane maintenance. Death was confirmed by the absence of a heartbeat, and corneal reflexes and paw withdrawal response to a noxious pinch. Ventricular tissue was subsequently dissected, washed, minced and subjected to three digestions at $37^{\circ} \mathrm{C}$ for $20 \mathrm{~min}$ in a solution containing a mixture of $1 \mathrm{mg} / \mathrm{ml}$ collagenase A and $0.5 \mathrm{mg} / \mathrm{ml}$ hyaluronidase following an initial digestion step in a proteinase bacterial solution $(4 \mathrm{U} / \mathrm{ml})$ for $15 \mathrm{~min}$. After each cycle of digestion, the tissue was mechanically dissociated using a $5 \mathrm{ml}$ pipette (Eppendorf), the dissociated cells were collected and resuspended in Dulbecco's modified Eagle's medium (DMEM; Gibco; Thermo Fisher Scientific, Inc.). CFs were separated from the cardiomyocytes by centrifugation $(1,000 \mathrm{x} \mathrm{g})$ at $4^{\circ} \mathrm{C}$ for $5 \mathrm{~min}$ and cultured to confluence in $10-\mathrm{cm}$ cell culture dishes in DMEM supplemented with 10\% FBS (Gibco; Thermo Fisher Scientific, Inc.), $1 \%$ penicillin and $1 \%$ streptomycin, and maintained at $37^{\circ} \mathrm{C}$ in a humidified atmosphere with $5 \% \mathrm{CO}_{2}$ and $95 \% \mathrm{O}_{2}$. CFs from the second passage were used for the subsequent experiments.

In the hypoxic group, CFs were exposed to 0,3 or $5 \%$ oxygen in an incubator connected with a chamber that was equilibrated with a water-saturated gas mixture of 0,3 or $5 \% \mathrm{O}_{2}, 5 \% \mathrm{CO}_{2}$ and 95,92 or $90 \% \mathrm{~N}_{2}$, respectively, at $37^{\circ} \mathrm{C}$ for 12,24 or $48 \mathrm{~h}$. In the normoxic group, CFs were exposed to $5 \% \mathrm{CO}_{2}$ and $95 \% \mathrm{O}_{2}$.

$C F$ transfection with miR-1 antagomiR. Negative control (NC) antagomiR and miR-1 antagomiR were synthesized by Guangzhou RiboBio Co., Ltd. CFs were seeded into 12-well plates at a density of $5 \times 10^{4}$ cells $/ \mathrm{ml}$ and transfected with $100 \mathrm{nM}$ $\mathrm{NC}$ antagomiR or miR-1 antagomiR using Lipofectamine 3,000 reagent (Invitrogen; Thermo Fisher Scientific, Inc.) for $6 \mathrm{~h}$. Subsequently, the CFs were treated with hypoxic or normoxic for $24 \mathrm{~h}$. The sequences of the oligonucleotides were as follows: NC antagomiR, 5'-CAGUACUUUUGUGUAGUACAA-3'; and miR-1 antagomiR, 5'-AUACAUACUUCUUUACAUUCCA-3'.

Masson's trichrome staining. The rats were sacrificed by cervical dislocation following anesthesia with $3.5 \%$ isoflurane induction and $2 \%$ isoflurane maintenance. and the hearts were removed. The RV tissues were fixed with $4 \%$ paraformaldehyde at $4^{\circ} \mathrm{C}$ for $24 \mathrm{~h}$, and embedded in paraffin. Cardiac sections $(5-\mu \mathrm{m})$ were subsequently analyzed using Masson's trichrome staining (Nanjing Biochannel Biotechnology Co., Ltd.) to measure the fibrosis of cardiomyocytes. Briefly, sections were incubated in celestine blue solution for $5 \mathrm{~min}$, washed with 
Table I. Primers used for reverse transcription-quantitative PCR.

\begin{tabular}{|c|c|c|c|}
\hline Gene & Species & Accession number & Primer sequence $\left(5^{\prime} \rightarrow 3^{\prime}\right)$ \\
\hline \multirow[t]{2}{*}{ Collagen I } & \multirow[t]{2}{*}{ Rat } & \multirow[t]{2}{*}{ BC133728 } & F: TCAAGATGGTGGCCGTTAC \\
\hline & & & R: CTGCGGATGTTCTCAATCTG \\
\hline \multirow[t]{2}{*}{ Collagen III } & \multirow[t]{2}{*}{ Rat } & \multirow[t]{2}{*}{ DN815278 } & F: CGAGATTAAAGCAAGAGGAA \\
\hline & & & R: GAGGCTTCTTTACATACCAC \\
\hline \multirow[t]{2}{*}{$\alpha$-smooth muscle actin } & \multirow[t]{2}{*}{ Rat } & \multirow[t]{2}{*}{$\mathrm{BC} 158550$} & F: GTCCCAGACATCAGGGAGTAA \\
\hline & & & R: TCGGATACTTCAGCGTCAGGA \\
\hline \multirow[t]{2}{*}{ Connective tissue growth factor } & \multirow[t]{2}{*}{ Rat } & \multirow[t]{2}{*}{ ВC072503 } & F: CAGGGAGTAAGGGACACGA \\
\hline & & & R: ACAGCAGTTAGGAACCCAGAT \\
\hline \multirow[t]{2}{*}{ miR-1 } & \multirow[t]{2}{*}{ Rat } & \multirow[t]{2}{*}{ NR032116 } & F: GGGGTGGAATGTAAAGAA \\
\hline & & & R: TGCGTGTCGTGGAGTC \\
\hline \multirow[t]{2}{*}{ U6 } & \multirow[t]{2}{*}{ Rat } & \multirow[t]{2}{*}{ K00784 } & F: GCTTCGGCAGCACATATACTAAAAT \\
\hline & & & R: CGCTTCACGAATTTGCGTGTCAT \\
\hline \multirow[t]{2}{*}{ GAPDH } & \multirow[t]{2}{*}{ Rat } & \multirow[t]{2}{*}{ AF106860 } & F: GGCACAGTCAAGGCTGAGAATG \\
\hline & & & R: ATGGTGGTGAAGACGCCAGTA \\
\hline
\end{tabular}

F, forward; R, reverse.

$\mathrm{H}_{2} \mathrm{O}$; incubated in hemalun solution for $5 \mathrm{~min}$, in $\mathrm{H}_{2} \mathrm{O}$ for $10 \mathrm{~min}$, in $0.5 \%$ fuchsine acid and $1.5 \%$ Ponceau xylidine for 5 min, washed with $\mathrm{H}_{2} \mathrm{O}$; incubated in $1 \%$ phosphomolybdic acid for $10 \mathrm{~min}$, in $2.5 \%$ aniline blue solution for $5 \mathrm{~min}$, washed with $\mathrm{H}_{2} \mathrm{O}$; incubated in $1 \%$ acetic acid for $1 \mathrm{~min}$, and then briefly in an ascending isopropanol series followed by xylol. All the operations were performed at room temperature. Then 3-5 randomly selected fields of view were selected from each of three sections from one rat and observed under a light microscope (magnification, x200; Carl Zeiss AG). Images were analyzed using Image-Pro Plus software (version 6.0; Media Cybernetics, Inc.).

Reverse transcription-quantitative PCR (RT-qPCR). Total RNA was extracted from RV tissues or CFs using TRIzol ${ }^{\circledR}$ reagent (Invitrogen; Thermo Fisher Scientific, Inc.). Total RNA was reverse transcribed into cDNA using random primers in a total volume of $10 \mu \mathrm{l}$ and PrimeScript ${ }^{\mathrm{TM}}$ RT Master mix (Takara Biotechnology Co., Ltd.), according to the manufacturer's protocol, at $37^{\circ} \mathrm{C}$ for $15 \mathrm{~min}$ and $85^{\circ} \mathrm{C}$ for $5 \mathrm{sec}$.cDNA was stored at $-70^{\circ} \mathrm{C}$ prior to use. qPCR of miR-1, collagen I, collagen III, $\alpha$-smooth muscle actin (SMA) and connective tissue growth factor (CTGF) expression levels were determined using SYBR Green I fluorescence (Invitrogen; Thermo Fisher Scientific, Inc). All samples were amplified in triplicate in 96-well plates using the following thermocycling conditions: Initial denaturation at $95^{\circ} \mathrm{C}$ for $10 \mathrm{~min}$; followed by 40 cycles at $95^{\circ} \mathrm{C}$ for $10 \mathrm{sec}$ and $60^{\circ} \mathrm{C}$ for $1 \mathrm{~min}$. GAPDH or U6 were used as the internal controls for mRNA and miRNA, respectively. Relative expression levels were quantified using the $2^{-\Delta \Delta \mathrm{Cq}}$ method (26-29). The primers used for the qPCR are shown in Table I.

Western blotting. Total protein was extracted from CFs using RIPA lysis buffer (BioChannel Biotechnology Co., Ltd.) and homogenized. Debris that had not been homogenized was removed, and the supernatant was obtained through centrifugation at $12,000 \times \mathrm{g}$ for $10 \mathrm{~min}$ at $4^{\circ} \mathrm{C}$. Total protein was quantified by BCA (Beyotime Institute of Biotechnology), and $\sim 50 \mu \mathrm{g}$ protein was separated via $8 \%$ SDS-PAGE. The separated proteins were subsequently transferred onto PVDF membranes and blocked by $5 \%$ skimmed milk powder at room temperature for $1 \mathrm{~h}$. The membranes were then incubated with the following primary antibodies at $4^{\circ} \mathrm{C}$ overnight: Anti-collagen I $(1: 2,000$; cat. no. ab34710; Abcam), anti-collagen III (1:5,000; cat. no. ab7778; Abcam), anti- $\alpha$-SMA (1:2,000; cat. no. ab32575; Abcam), anti-CTGF (1:1,000; cat. no. ab6992; Abcam), anti-PI3K (1:1,000; cat. no. 4249; Cell Signaling Technology, Inc.), anti-phosphorylated (p)-PI3K (1:1,000; cat. no. 17366; Cell Signaling Technology, Inc.), anti-AKT (1:1,000; cat. no. 4691; Cell Signaling Technology, Inc.), anti-p-AKT (1:2,000; cat. no. 4060; Cell Signaling Technology, Inc.) and anti-GAPDH (1:10,000, cat. no. ab181602; Abcam). The horseradish peroxidase-conjugated goat anti-rabbit secondary antibody (1:10,000, cat. no. ab7090; Abcam) was added and incubated at room temperature for $1 \mathrm{~h}$. ECL kit (Beyotime Institute of Biotechnology) was used to visualize the proteins. Densitometric analysis was performed using Image-Pro Plus software (version 6.0; Media Cybernetics, Inc.).

Statistical analysis. Statistical analysis was performed using GraphPad Prism 6.0 software (GraphPad Software, Inc.). Data are presented as the mean \pm SEM. Statistical differences between two groups were determined using a unpaired Student's t-test, while statistical differences between multiple groups were determined using a one-way ANOVA followed by a Bonferroni's post hoc test. A total of 3 experimental repeats were performed. $\mathrm{P}<0.05$ was considered to indicate a statistically significant difference.

\section{Results}

Hypoxia induces $P A H$ in rats. PAH was successfully induced by hypoxia in the rat model, as evidenced by an increased mPAP (Fig. 1A) and RVSP (Fig. 1B) compared with rats 

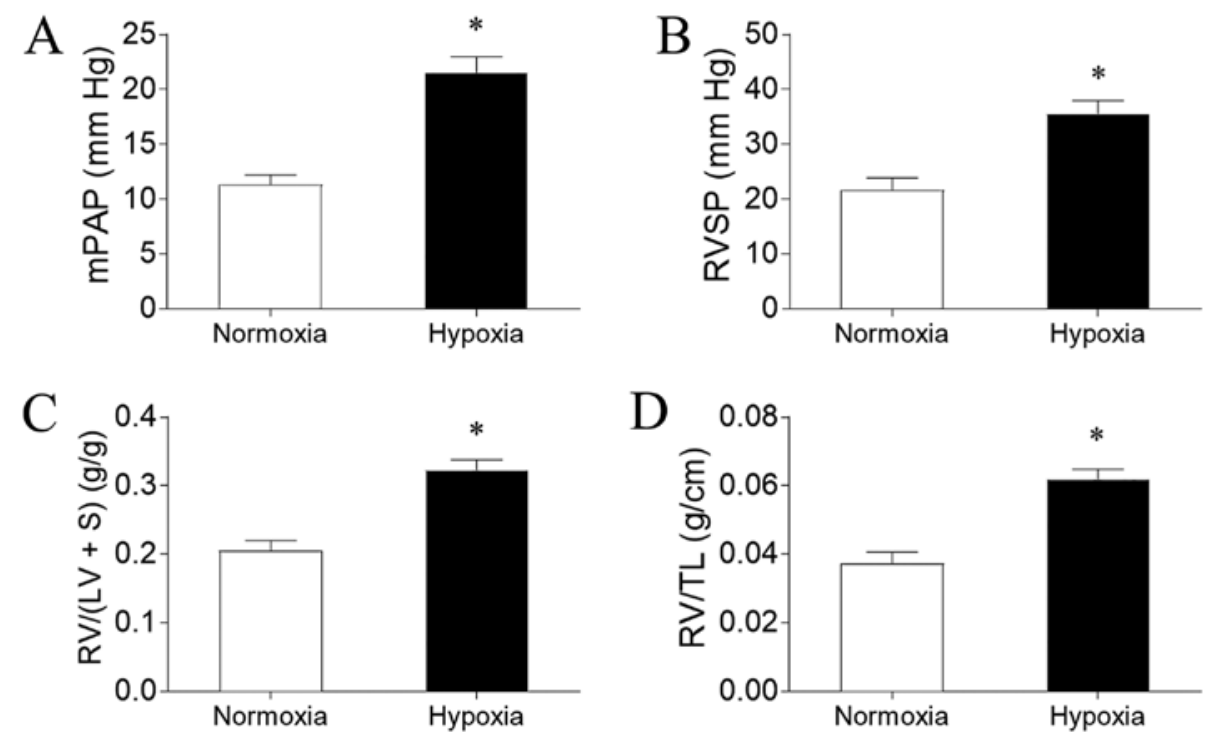

Figure 1. Establishment of PAH model rats through induction by hypoxia. (A) mPAP, (B) RVSP, (C) RV/(LV+S) (D) and RV/TL were increased in PAH model rats exposed to hypoxia. The results are presented as the mean \pm SEM. $n=8$ in normoxia group and $n=13$ in hypoxia group. "P<0.05 vs. normoxia. mPAP, mean pulmonary arterial pressure; RVSP, right ventricle systolic pressure; RV, right ventricle; LV, left ventricle; S, interventricular septum; TL, tibia length; PAH, pulmonary arterial hypertension.
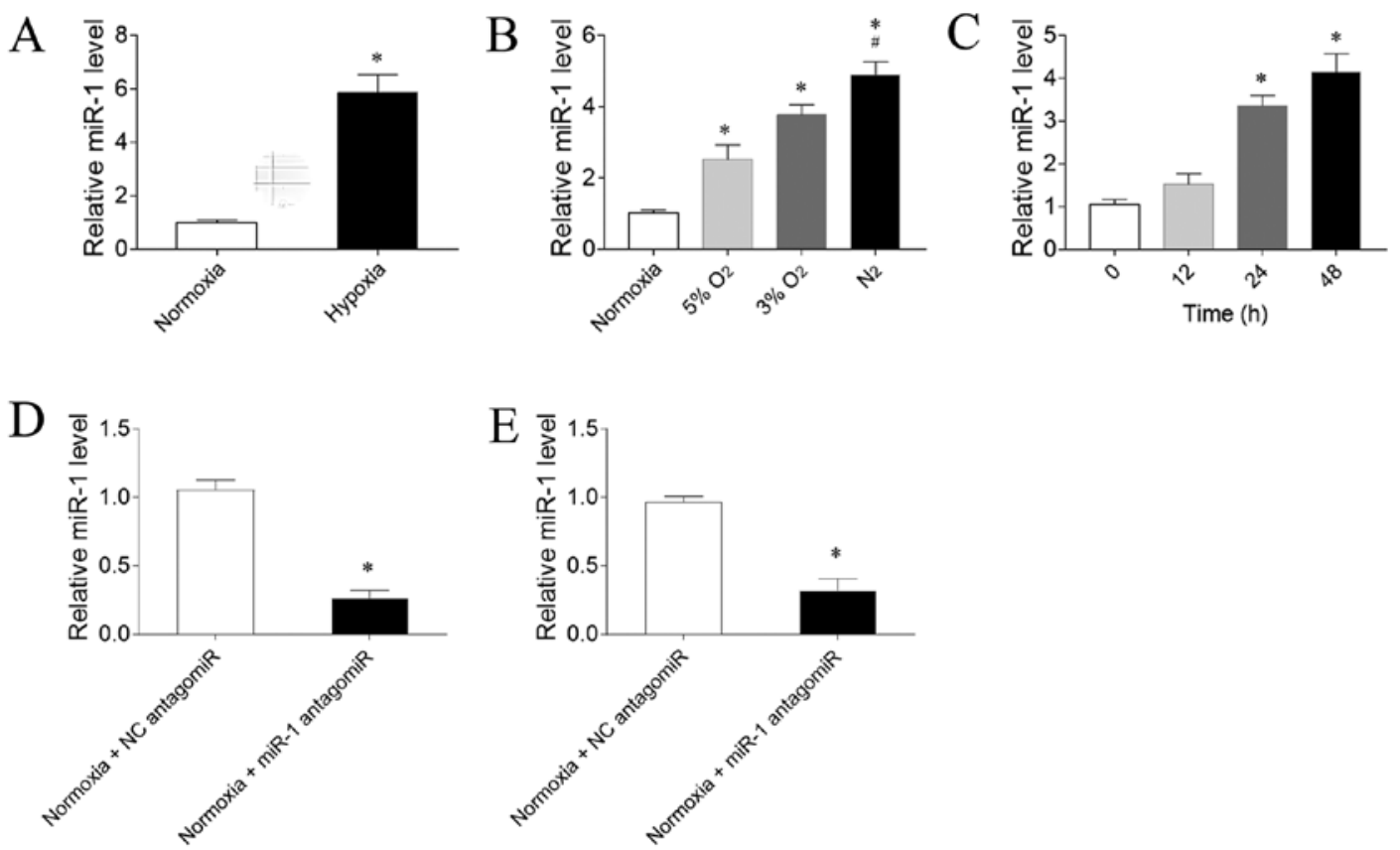

Figure 2. Expression levels of miR-1 in the RV of rats and CFs. (A) miR-1 expression levels were upregulated in RV of PAH model rats exposed to hypoxia. miR-1 expression levels were upregulated in CFs exposed to (B) hypoxia and (C) hypoxia for different durations. (D) miR-1 antagomiR transfection significantly downregulated the expression levels of miR-1 in the RV of PAH model rats. (E) miR-1 antagomiR transfection significantly downregulated the expression levels of miR-1 in CFs. The results are presented as the mean \pm SEM. $n=8$ in normoxia group and $n=13$ in hypoxia group. ${ }^{*} \mathrm{P}<0.05 \mathrm{vs}$. normoxia/0 $\mathrm{h} /$ normoxia + $\mathrm{NC}$ antagomiR; ${ }^{\mathrm{P}} \mathrm{P}<0.05$ vs. $5 \% \mathrm{O}_{2}$. miR, microRNA; PAH, pulmonary arterial hypertension; $\mathrm{CFs}$, cardiac fibroblasts; $\mathrm{NC}$, negative control.

exposed to normoxia. Hypoxia exposure also significantly increased RV/(LV + S) (Fig. 1C) and RV/TL (Fig. 1D) in the rats compared with normoxia exposure.

Expression levels of miR-1. The expression levels of miR-1 were significantly increased in the RV of PAH model rats exposed to hypoxia compared with rats exposed to normoxia (Fig. 2A). To determine the effect of hypoxia on the expression levels of miR-1 in CFs, three gradient $\mathrm{O}_{2}$ concentrations were used. The expression levels of miR-1 in CFs were sequentially upregulated as the $\mathrm{O}_{2}$ concentration gradually decreased compared with the normoxia group; the exposure to 5 or $3 \% \mathrm{O}_{2}$ significantly upregulated miR-1 expression levels compared with exposure to normoxia. Notably, exposure to $\mathrm{N}_{2}$ was more powerful in upregulating miR-1 expression levels compared with 5\% $\mathrm{O}_{2}$ exposure (Fig. 2B). $3 \% \mathrm{O}_{2}$ was selected for use in the following experiments. The expression levels of miR-1 were significantly upregulated following $24 \mathrm{~h}$, but not 

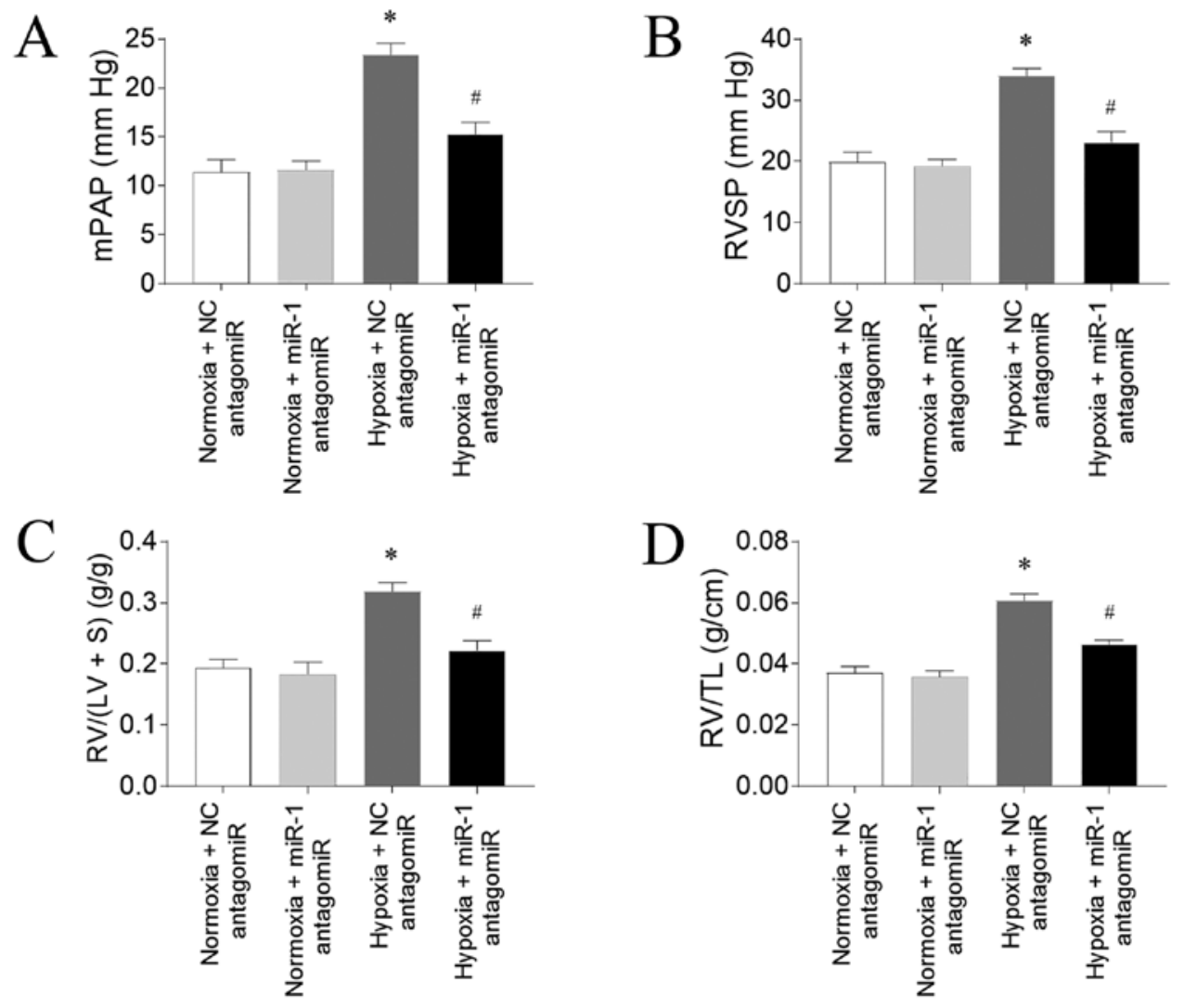

Figure 3. miR-1 antagomiR transfection attenuates hypoxia-induced PAH in rats. miR-1 antagomiR transfection inhibited the increases in the (A) mPAP, (B) RVSP, (C) RV/(LV+S) and (D) RV/TL in PAH model rats exposed to hypoxia. The results are presented as the mean \pm SEM. $n=10$ in normoxia $+\mathrm{NC}$ antagomiR and normoxia + miR-1 antagomiR groups and $n=15$ in hypoxia $+\mathrm{NC}$ antagomiR and hypoxia + miR-1 antagomiR groups. ${ }^{*} \mathrm{P}<0.05$ vs. normoxia $+\mathrm{NC}$ antagomiR; ${ }^{\text {P }}<0.05$ vs. hypoxia $+\mathrm{NC}$ antagomiR. miR, microRNA; PAH, pulmonary arterial hypertension; mPAP, mean pulmonary arterial pressure; RVSP, right ventricle systolic pressure; RV, right ventricle; LV, left ventricle; S, interventricular septum; TL, tibia length; NC, negative control.

$12 \mathrm{~h}$, of hypoxia exposure compared with CFs not exposed to hypoxia; however, this upregulation was not further enhanced after $48 \mathrm{~h}$ of exposure compared with $24 \mathrm{~h}$ (Fig. 2C). Thus, $24 \mathrm{~h}$ hypoxia stimulation was used in the following in vitro experiments. miR-1 antagomiR significantly downregulated the expression levels of miR-1 in the RV of rats compared with the NC antagomiR (Fig. 2D). Furthermore, the expression levels of miR-1 were significantly downregulated in CFs transfected with miR-1 antagomiR compared with NC antagomiR (Fig. 2E).

Effects of miR-1 antagomiR on PAH. Hypoxia-induced an increase in mPAP, which was inhibited by miR-1 antagomiR (Fig. 3A). RVSP was increased in the rats treated with hypoxia, which was reversed by miR antagomiR (Fig. 3B). The increase of $\mathrm{RV} /(\mathrm{LV}+\mathrm{S})$ of rats induced by hypoxia was alleviated by miR antagomiR administration (Fig. 3C). RV/TL was elevated in the rats treated by hypoxia, and this increase was attenuated by administration of miR-1 antagomiR (Fig. 3D).

Effects of miR-1 antagomiR on fibrosis in PAH model rats. According to the results of Masson's trichrome staining, the RV fibrosis was increased following hypoxia treatment; this increase was subsequently partially reversed following miR-1 antagomiR transfection (Fig. 4A). The mRNA expression levels of collagen I, collagen III, $\alpha$-SMA and CTGF in the $\mathrm{RV}$ of PAH model rats exposed to hypoxia were significantly upregulated; these increases were partially inhibited following miR-1 antagomiR transfection (Fig. 4B).

Effects of miR-1 antagomiR on fibrosis in CFs. Following $3 \% \mathrm{O}_{2}$ exposure (hypoxia), the mRNA expression levels of collagen I, collagen III, $\alpha$-SMA and CTGF were significantly upregulated in CFs compared with the normoxia group, which were all subsequently attenuated following miR-1 antagomiR transfection (Fig. 5A). The protein expression levels of collagen I, collagen III, $\alpha$-SMA and CTGF were also significantly upregulated in $\mathrm{CFs}$ exposed to hypoxia compared with the normoxia group, and these increases were partially inhibited by miR-1 antagomiR transfection (Fig. 5B).

Effects of miR-1 antagomiR on fibrosis in CFs. The expression levels of collagen I in CFs isolated from PAH model rats (PCFs) were significantly upregulated compared with $\mathrm{CFs}$ isolated from normoxia rats (NCFs), while the subsequent transfection with miR-1 antagomiR inhibited this upregulation (Fig. 6). Collagen III expression levels were also significantly upregulated in PAFs compared with NCFs, and were also attenuated by miR-1 antagomiR transfection. Similarly, the expression levels of $\alpha$-SMA and CTGF in PCFs treated with NC antagomiR were upregulated compared with in NCFs treated with NC antagomiR, and the increases in $\alpha$-SMA and CTGF expression levels in PCFs were inhibited by miR-1antagomiR transfection. 


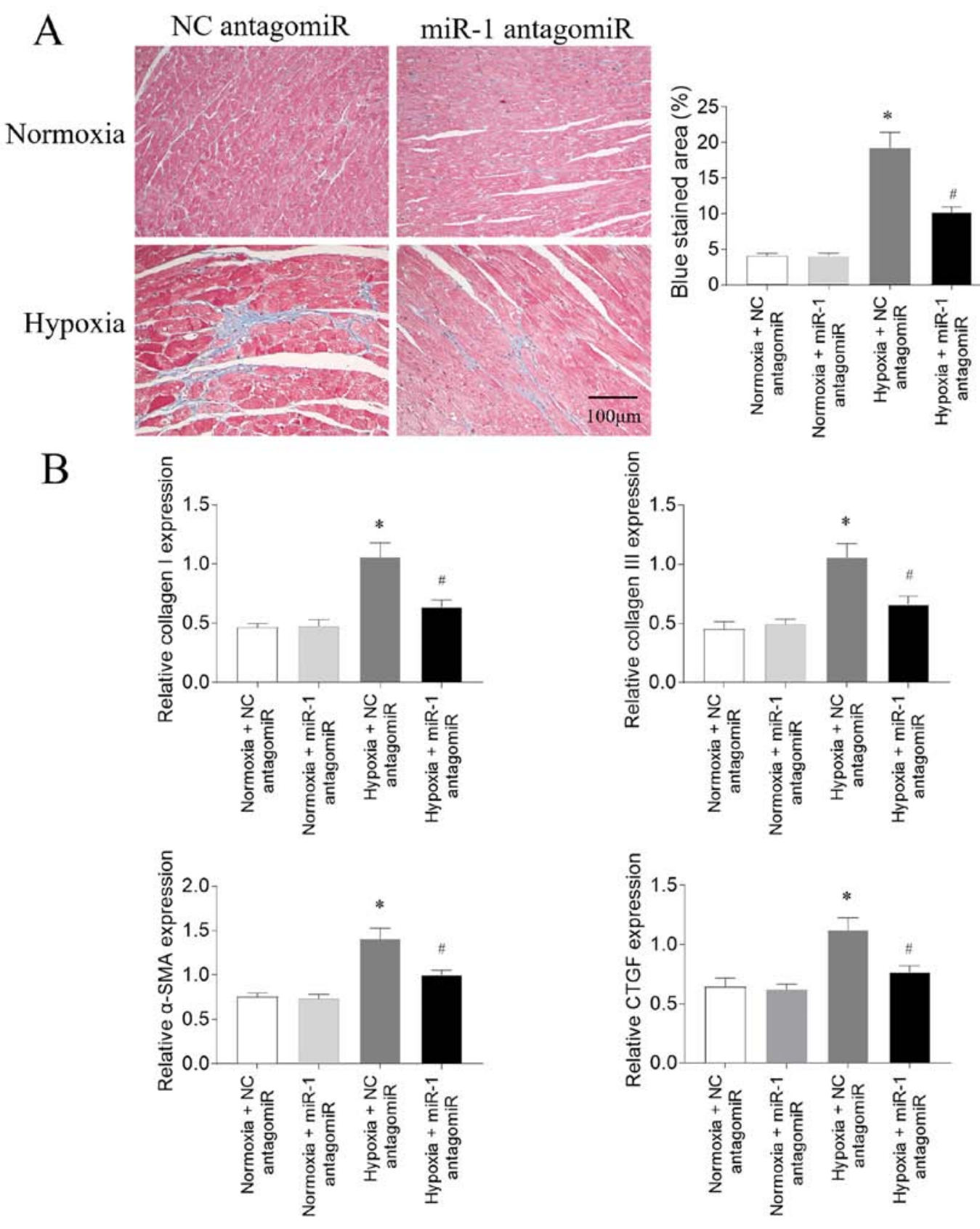

Figure 4. miR-1 antagomiR attenuates RV fibrosis in hypoxia-induced PAH model rats. (A) miR-1 antagomiR transfection inhibits the increase in RV fibrosis in PAH model rats exposed to hypoxia. (B) miR-1 antagomiR transfection inhibits the upregulated expression levels of collagen I, collagen III, $\alpha$-SMA and CTGF in the RV of PAH model rats exposed to hypoxia. The results are presented as the mean \pm SEM. $n=10$ in the normoxia $+\mathrm{NC}$ antagomiR and normoxia + miR-1 antagomiR groups and $\mathrm{n}=15$ in the hypoxia $+\mathrm{NC}$ antagomiR and hypoxia $+\mathrm{miR}-1$ antagomiR groups. ${ }^{*} \mathrm{P}<0.05 \mathrm{vs.}$ normoxia $+\mathrm{NC}$ antagomiR; ${ }^{\text {"P}} \mathrm{P}<0.05$ vs. hypoxia $+\mathrm{NC}$ antagomiR. Scale bar, $100 \mu \mathrm{m}$. miR, microRNA; RV, right ventricle; PAH, pulmonary arterial hypertension; SMA, smooth muscle actin; CTGF, connective tissue growth factor; NC, negative control.

Involvement of the PIBK/AKT signaling pathway in PAH. The expression levels of p-PI3K/PI3K were upregulated in CFs exposed to hypoxia, and this increase was subsequently inhibited by miR-1 antagomiR transfection. Furthermore, the expression levels of p-AKT were also upregulated in CFs exposed to hypoxia compared with the normoxia $\mathrm{CFs}$, and this increase was partially reversed following miR-1 antagomiR transfection (Fig. 7).

\section{Discussion}

Hypoxia plays an initiating role in the pathogenesis of PAH. Numerous miRs have been found to be dysregulated in the lung and heart of PAH model rats under chronic hypoxic and monocrotaline (MCT) environments (30-32). The results of the present study demonstrated that knocking down miR-1 expression attenuated PAH and RV fibrosis in PAH model rats, a process that was suggested to involve the PI3K/AKT signaling pathway.

PAH is refractory and devastating; however, there are currently no effective treatments available. miRNAs have emerged as novel targets for PAH treatment and numerous miRNAs play a rolein the development of PAH(33). For example, one previous study reported that miR-204 expression levels were downregulated in lung tissue from humans, mice and rats 
A
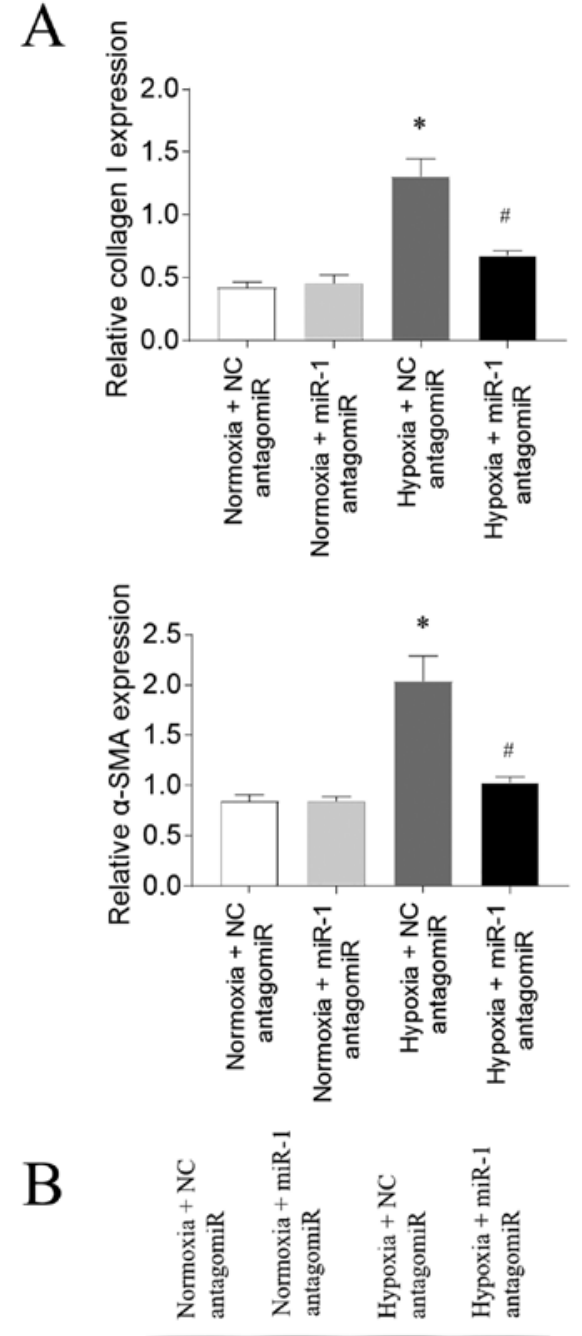

Collagen I

Collagen III

$\alpha-\mathrm{SMA}$

CTGF

GAPDH
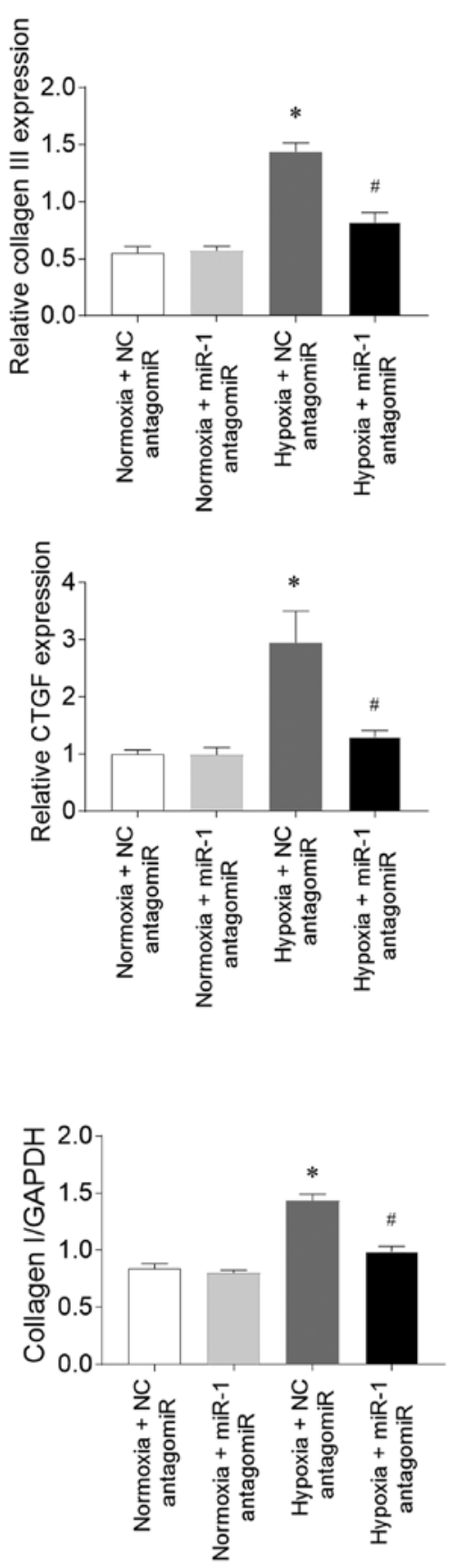

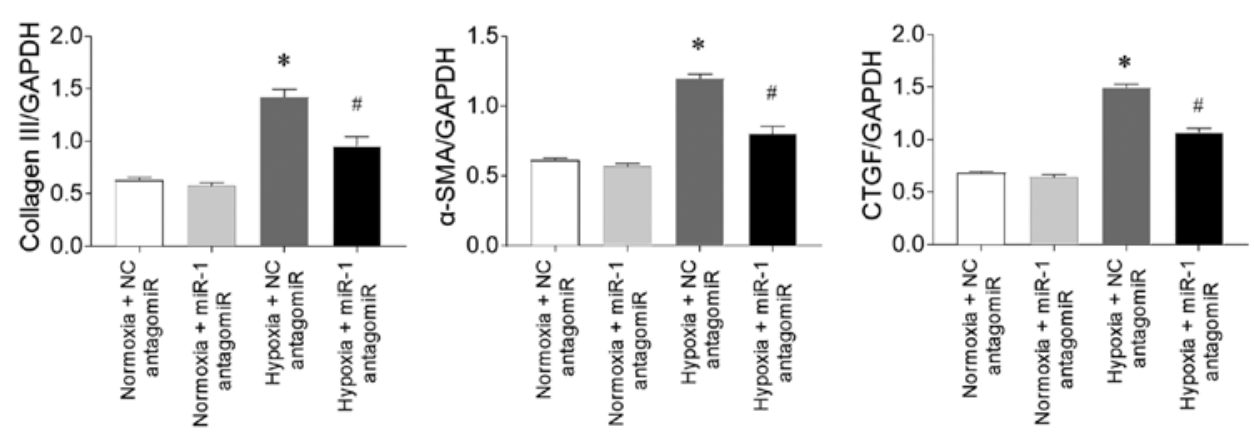

Figure 5. miR-1 antagomiR attenuates the fibrosis of CFs induced by hypoxia. miR-1 antagomiR transfection inhibited the upregulation in the (A) mRNA and (B) protein expression levels of collagen I, collagen III, $\alpha$-SMA and CTGF in CFs induced by hypoxia. The results are presented as mean \pm SEM. ${ }^{*} \mathrm{P}<0.05$ vs. normoxia + NC antagomiR; ${ }^{\#} \mathrm{P}<0.05$ vs. hypoxia + NC antagomiR. miR, microRNA; CFs, cardiac fibroblasts; SMA, smooth muscle actin; $\mathrm{CTCF}$, connective tissue growth factor; NC, negative control.

with PAH, and knocking down miR-204 expression increased the proliferation and decreased the apoptosis of pulmonary artery smooth muscle cells in patients with PAH (34). The expression levels of miRNAs show model-derived differences. 

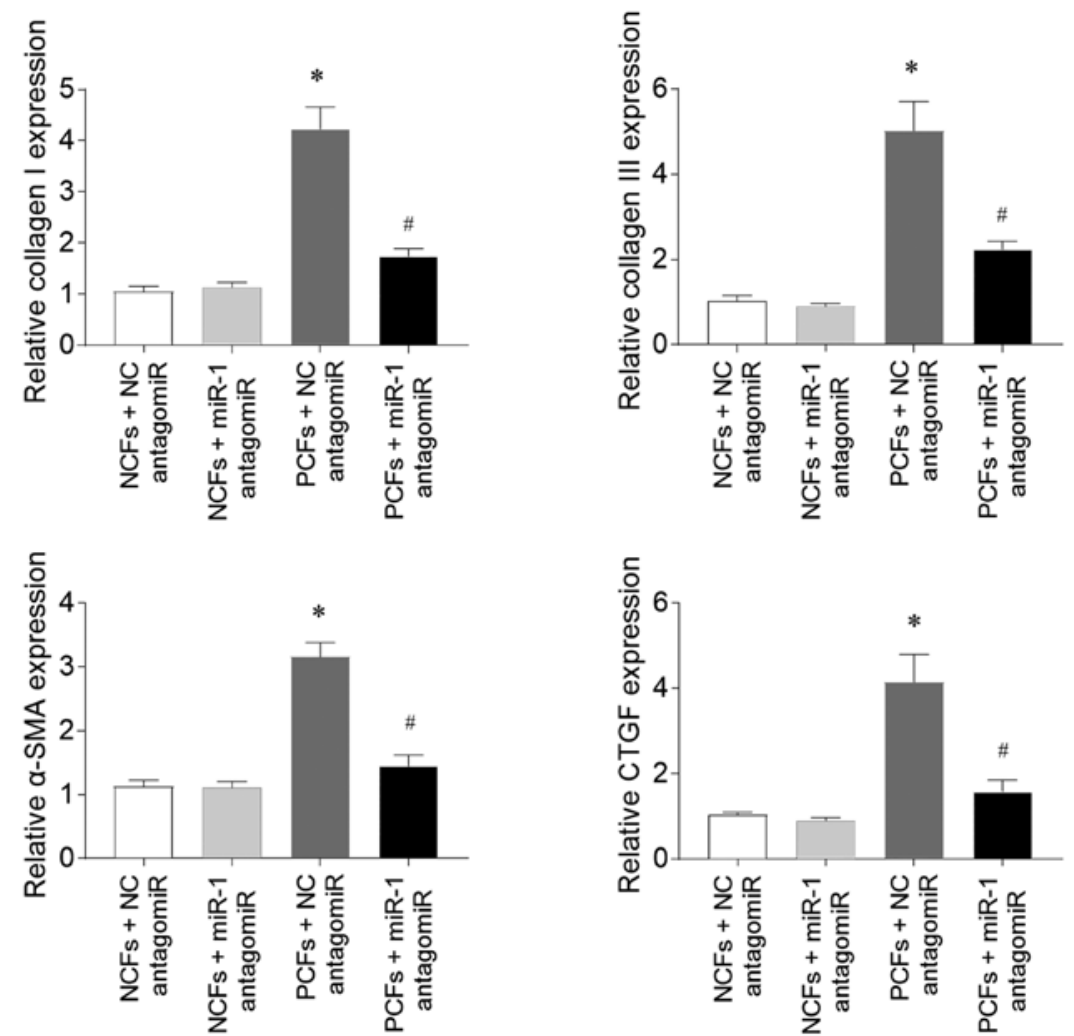

Figure 6. miR-1 antagomiR attenuates the fibrosis in CFs isolated from PAH model rats. The expression levels of collagen I, collagen III, $\alpha$-SMA and CTGF in PCFs treated with NC antagomiR were upregulated compared with NCFs treated with NC antagomiR. These increases were subsequently inhibited by transfection with the miR-1 antagomiR. The results are presented as the mean $\pm \mathrm{SEM}$. ${ }^{*} \mathrm{P}<0.05$ vs. normoxia $+\mathrm{NC}$ antagomiR; ${ }^{\sharp} \mathrm{P}<0.05$ vs. hypoxia $+\mathrm{NC}$ antagomiR. miR, microRNA; CFs, cardiac fibroblasts; PAH, pulmonary arterial hypertension; SMA, smooth muscle actin; CTGF, connective tissue growth factor; NC, negative control; PCFs, $\mathrm{CFs}$ isolated from PAH model rats; NCFs, CFS isolated from normoxia rats.

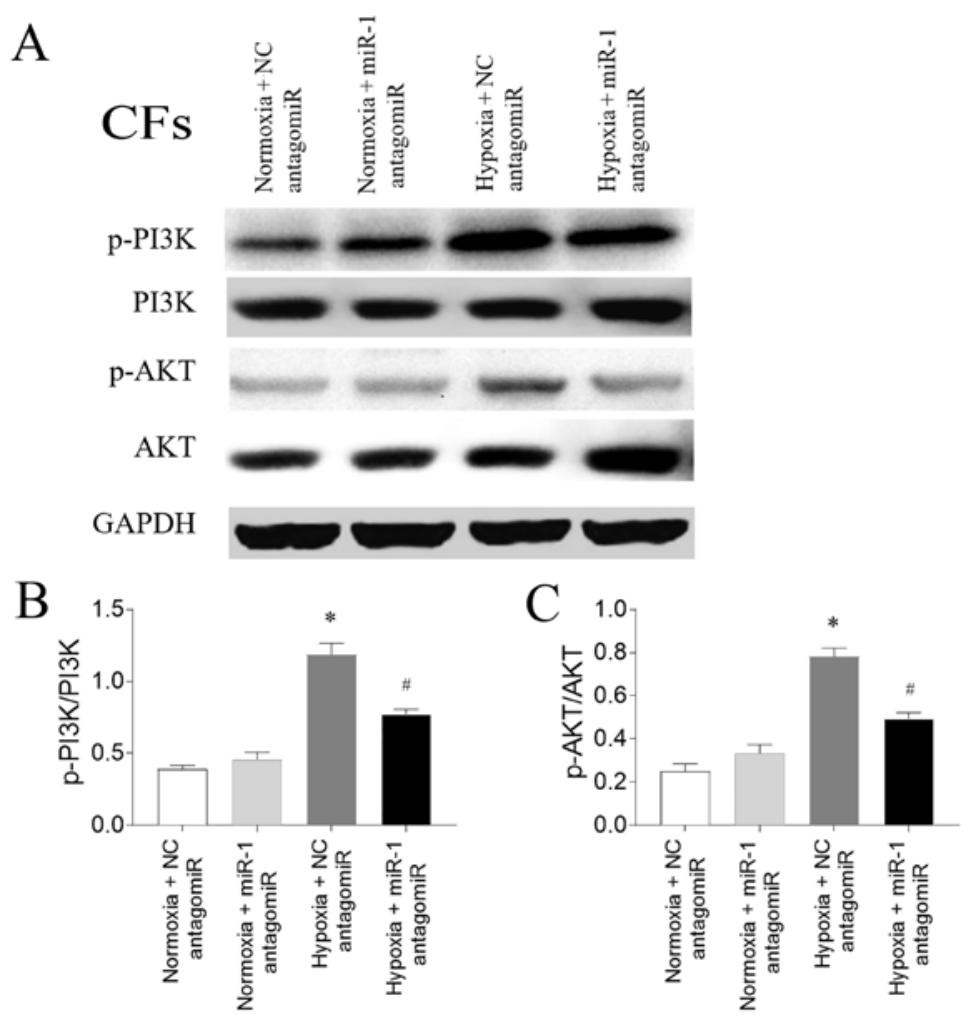

Figure 7. Enhancement of PI3K/AKT signaling pathway is inhibited by miR-1 antagomiR. (A) Representative western blotting of p-PI3K, PI3K, p-AKT and AKT expression levels. Expression levels of (B) p-PI3K/PI3K and (C) p-AKT/AKT were upregulated in CFs induced by hypoxia, and this increase was reversed by transfection with miR-1 antagomiR. The results are presented as the mean $\pm \mathrm{SEM}$. ${ }^{*} \mathrm{P}<0.05$ vs. normoxia $+\mathrm{NC}$ antagomiR; ${ }^{*} \mathrm{P}<0.05$ vs. hypoxia $+\mathrm{NC}$ antagomiR. miR, microRNA; p-, phosphorylated; $\mathrm{NC}$, negative control; $\mathrm{CFs}$, cardiac fibroblasts. 
For example, MCT and hypoxia induced consistent changes in miR-30c and miR-451, yet regulated miR-22 and miR-21 differently, suggesting that hypoxia- and MCT-induced PAH share some common elements relating to miRs regulation and differential regulation on miRs (31). The results of the present study revealed that the expression levels of miR-1 in the RV were upregulated in rats with hypoxia-induced PAH. In PAH model rats, mPAP and RVSP were also increased, while knocking down miR-1 expression with an antagomiR reversed these increases in PAH model rats. These results suggested that the expression levels of miR-1 may be dysregulated in the RV of PAH model rats, and that knocking down miR-1 expression may significantly attenuate PAH.

PAH exerts significant pressure on the RV, usually resulting in RV remodeling (35). Pathological hypertrophy is a feature of RV remodeling (36). Restoring the expression of miR-223 in the lungs of rats with MCT-induced PAH provided beneficial effects on RV hypertrophy and vascular remodeling in a previous study (37). In the present study, RV hypertrophy was increased in PAH model rats exposed to hypoxia, as indicated by the increases in the RV/(LV+S) and RV/TL. Transfection with the miR-1 antagomiR reversed these increases, indicating that knocking down miR-1 may control RV hypertrophy in PAH model rats.

$\mathrm{RV}$ fibrosis is another feature of $\mathrm{PAH}$-induced RV remodeling, which is consistently observed in patients with PAH $(38,39)$ and animal models $(40,41)$. The present study found that the expression levels of collagen I, collagen III, $\alpha$-SMA and CTGF were upregulated in the RV of PAH model rats exposed to normoxia, and these increases were inhibited following miR-1 antagomiR transfection. miR-1 antagomiR also attenuated the increases in the expression levels of collagen I, collagen III, $\alpha$-SMA and CTGF in CFs stimulated with hypoxia. Similarly, the expression levels of collagen I, collagen III, $\alpha$-SMA and CTGF in CFs from PAH model rats were upregulated, which were downregulated by miR-1 antagomiR transfection. These results suggested that knocking down miR-1 expression may reverse the fibrosis of $\mathrm{RV}$ in PAH model rats.

The PI3K/AKT signaling pathway plays a key role in the fibrosis of the heart (42). Cardiac fibroblast proliferation and migration following myocardial infarction were found to be regulated by the PTEN/PI3K/AKT/mTOR signaling pathway (20). PI3K/AKT signaling was also demonstrated to be necessary for hypoxia-induced CF differentiation and extracellular matrix synthesis (43). The results of the present study reported that the expression levels of p-PI3K were upregulated in CFs exposed with hypoxia, while the transfection with the miR-1 antagomiR partially inhibited this increase. Furthermore, the expression levels of p-AKT were also upregulated in CFs exposed with hypoxia, and these expression levels were also reversed following miR-1 antagomiR transfection. These results indicated that the PI3K/AKT signaling pathway may be involved in the regulation of miR-1 in the cardiac fibrosis of PAH.

In conclusion, the findings of the present study suggested that knocking down miR-1 expression may control PAH, and attenuate RV hypertrophy and fibrosis induced by PAH. The results indicated that these effects may occur via a regulatory mechanism that may involve the PI3K/AKT signaling pathway.
Future studies should aim to analyze the expression levels of miR-1 in patients with PAH to determine whether miR-1 expression is dysregulated. The present results suggested that miR-1 may be a potential novel target for the treatment of PAH.

\section{Acknowledgements}

Not applicable

\section{Funding}

The present study was supported by grants from the Priority Academic Program Development of Jiangsu Higher Education Institutions (PAPD).

\section{Availability of data and materials}

The datasets used and/or analyzed during the current study are available from the corresponding author on reasonable request.

\section{Authors' contributions}

$\mathrm{Yu} \mathrm{L}$ and Yo L made substantial contributions to conception and design, and acquisition of data. JL and $\mathrm{XZ}$ made substantial contributions to analysis of data. QC and WX made substantial contributions to interpretation of data. HW made substantial contributions to design, and drafting the manuscript and revising it. Yu L and HW were responsible for confirming the authenticity of raw data. All authors reviewed and approved the final manuscript.

\section{Ethics approval and consent to participate}

All procedures were approved by the Experimental Animal Care and Use Committee of Nanjing Medical University (Nanjing, China; approval no. 17041015), and were conducted in accordance with the Guide for the Care and Use of Laboratory Animals (National Institutes of Health publication no. 85-23, revised 1996) (22).

\section{Patient consent for publication}

Not applicable.

\section{Competing interests}

The authors declare that they have no competing interests.

\section{References}

1. Kovacs G, Dumitrescu D, Barner A, Greiner S, Grünig E, Hager A, Köhler T, Kozlik-Feldmann R, Kruck I, Lammers AE, Mereles D, et al: Definition, clinical classification and initial diagnosis of pulmonary hypertension: Updated recommendations from the cologne consensus conference 2018. Int J Cardiol 272S: 11-19, 2018.

2. Lan NSH, Massam BD, Kulkarni SS and Lang CC: Pulmonary arterial hypertension: Pathophysiology and treatment. Diseases 6: 38, 2018.

3. Guiot J, Parzibut G, Weber T, Davin L, Dulgheru R, Lancellotti P, Louis R and Vachiery JL: Pulmonary arterial hypertension. Rev Med Liege 74: 139-45, 2019 (In French). 
4. Thenappan T, Ormiston ML, Ryan JJ and Archer SL: Pulmonary arterial hypertension: Pathogenesis and clinical management. BMJ 360: j5492, 2018.

5. Bartel DP: MicroRNAs: Genomics, biogenesis, mechanism, and function. Cell 116: 281-297, 2004.

6. Javadian M, Gharibi T, Shekari N, Abdollahpour-Alitappeh M, Mohammadi A, Hossieni A, Mohammadi H and Kazemi T: The role of microRNAs regulating the expression of matrix metalloproteinases (MMPs) in breast cancer development, progression, and metastasis. J Cell Physiol 234: 5399-5412, 2019.

7. van Rooij E: The art of microRNA research. Circ Res 108: 219-234, 2011.

8. Mohr AM and Mott JL: Overview of microRNA biology. Semin Liver Dis 35: 3-11, 2015.

9. Dawson K, Wakili R, Ordog B, Clauss S, Chen Y, Iwasaki Y, Voigt N, Qi XY, Sinner MF, Dobrev D, et al: MicroRNA29: A mechanistic contributor and potential biomarker in atrial fibrillation. Circulation 127: 1466-1475, 1475e1-28, 2013.

10. Eitel I, Adams V, Dieterich P, Fuernau G, de Waha S, Desch S, Schuler G and Thiele H: Relation of circulating MicroRNA-133a concentrations with myocardial damage and clinical prognosis in ST-elevation myocardial infarction. Am Heart J 164: 706-714, 2012.

11. Deng L, Blanco FJ, Stevens H, Lu R, Caudrillier A, McBride M, McClure JD, Grant J, Thomas M, Frid M, et al: MicroRNA-143 activation regulates smooth muscle and endothelial cell crosstalk in pulmonary arterial hypertension. Circ Res 117: 870-883, 2015

12. Caruso P, Dunmore BJ, Schlosser K, Schoors S, Dos Santos C, Perez-Iratxeta C, Lavoie JR, Zhang H, Long L, Flockton AR, et al: Identification of MicroRNA-124 as a major regulator of enhanced endothelial cell glycolysis in pulmonary arterial hypertension via PTBP1 (Polypyrimidine Tract Binding Protein) and pyruvate kinase M2. Circulation 136: 2451-2467, 2017.

13. Rothman AM, Arnold ND, Pickworth JA, Iremonger J, Ciuclan L, Allen RM, Guth-Gundel S, Southwood M, Morrell NW, Thomas M, et al: MicroRNA-140-5p and SMURF1 regulate pulmonary arterial hypertension. J Clin Invest 126: 2495-2508, 2016.

14. Lee HW and Park SH: Elevated microRNA-135a is associated with pulmonary arterial hypertension in experimental mouse model. Oncotarget 8: 35609-35618, 2017.

15. Karakikes I, Chaanine AH, Kang S, Mukete BN, Jeong D, Zhang S, Hajjar RJ and Lebeche D: Therapeutic cardiac-targeted delivery of miR-1 reverses pressure overload-induced cardiac hypertrophy and attenuates pathological remodeling. J Am Heart Assoc 2: e000078, 2013.

16. Yin H, Zhao L, Zhang S, Zhang $\mathrm{Y}$ and Lei S: MicroRNA1 suppresses cardiac hypertrophy by targeting nuclear factor of activated T cells cytoplasmic 3. Mol Med Rep 12: 8282-8288, 2015.

17. Lapikova-Bryhinska T, Zhukovska A, Nagibin V, Tumanovska L, Portnichenko G, Goncharov S, Portnychenko A and Dosenko V: Altered biogenesis of microRNA-1 is associated with cardiac dysfunction in aging of spontaneously hypertensive rats. Mol Cell Biochem 459: 73-82, 2019.

18. Mondejar-Parreno G, Callejo M, Barreira B, Morales-Cano D, Esquivel-Ruiz S, Filice M, Moreno L, Cogolludo A and PerezVizcaino F: miR-1 induces endothelial dysfunction in rat pulmonary arteries. J Physiol Biochem 75: 519-529, 2019.

19. Wei L,Zhang Y,Qi X, Sun X,Li Y and Xu Y: Ubiquitinproteasomes are the dominant mediators of the regulatory effect of microRNA1 on cardiac remodeling after myocardial infarction. Int J Mol Med 44: 1899-1907, 2019.

20. Yang W, Wu Z, Yang K, Han Y, Chen Y, Zhao W, Huang F, Jin Y and Jin W: BMI1 promotes cardiac fibrosis in ischemia-induced heart failure via the PTEN-PI3K/Akt-mTOR signaling pathway. Am J Physiol Heart Circ Physiol 316: H61-H69, 2019.

21. Zhang CJ, Huang Y, Lu JD, Lin J, Ge ZR and Huang $H$ : Upregulated microRNA-132 rescues cardiac fibrosis and restores cardiocyte proliferation in dilated cardiomyopathy through the phosphatase and tensin homolog-mediated PI3K/Akt signal transduction pathway. J Cell Biochem: Sep 14, 2018 (Epub ahead of print). doi: $10.1002 /$ jcb. 27081

22. National Research Council: Guide for the Care and Use of Laboratory Animals, National Academies Press, Washington, DC, 1996.

23. Luo H, Liu B, Zhao L, He J, Li T, Zha L, Li X, Qi Q, Liu Y and Yu Z: Galectin-3 mediates pulmonary vascular remodeling in hypoxia-induced pulmonary arterial hypertension. J Am Soc Hypertens 11: 673-683.e3, 2017.
24. Wang Y, Pandey RN, York AJ, Mallela J, Nichols WC, Hu YC, Molkentin JD, Wikenheiser-Brokamp KA and Hegde RS: The EYA3 tyrosine phosphatase activity promotes pulmonary vascular remodeling in pulmonary arterial hypertension. Nat Commun 10: 4143, 2019.

25. Luo Y, Teng X, Zhang L, Chen J, Liu Z, Chen X, Zhao S, Yang S, Feng J and Yan X: CD146-HIF-1alpha hypoxic reprogramming drives vascular remodeling and pulmonary arterial hypertension. Nat Commun 10: 3551, 2019

26. Zhao H, Ma TF, Lin J, Liu LL, Sun WJ, Guo LX, Wang SQ, Otecko NO and Zhang YP: Identification of valid reference genes for mRNA and microRNA normalisation in prostate cancer cell lines. Sci Rep 8: 1949, 2018.

27. Peltier HJ and Latham GJ: Normalization of microRNA expression levels in quantitative RT-PCR assays: Identification of suitable reference RNA targets in normal and cancerous human solid tissues. Rna 14: 844-852, 2008.

28. Inada K, Okoshi Y, Cho-Isoda Y, Ishiguro S, Suzuki H, Oki A, Tamaki Y, Shimazui T, Saito H, Hori M, et al: Endogenous reference RNAs for microRNA quantitation in formalin-fixed, paraffin-embedded lymph node tissue. Sci Rep 8: 5918, 2018.

29. Livak KJ and Schmittgen TD: Analysis of relative gene expression data using real-time quantitative PCR and the 2(-Delta Delta C(T)) method. Methods 25: 402-408, 2001.

30. Zhu Z, Fang Z, Hu X and Zhou S: MicroRNAs and mesenchymal stem cells: Hope for pulmonary hypertension. Rev Bras Cir Cardiovasc 30: 380-385, 2015.

31. Caruso P, MacLean MR, Khanin R, McClure J, Soon E, Southgate M, MacDonald RA, Greig JA, Robertson KE, Masson R, et al: Dynamic changes in lung microRNA profiles during the development of pulmonary hypertension due to chronic hypoxia and monocrotaline. Arterioscler Thromb Vasc Biol 30: 716-723, 2010.

32. Zhou SS, Jin JP, Wang JQ, Zhang ZG, Freedman JH, Zheng Y and Cai L: miRNAS in cardiovascular diseases: Potential biomarkers, therapeutic targets and challenges. Acta Pharmacol Sin 39: 1073-1084, 2018.

33. Zhou G, Chen T and Raj JU: MicroRNAs in pulmonary arterial hypertension. Am J Respir Cell Mol Biol 52: 139-151, 2015.

34. Courboulin A, Paulin R, Giguere NJ, Saksouk N, Perreault T, Meloche J, Paquet ER, Biardel S, Provencher S, Côté J, et al: Role for miR-204 in human pulmonary arterial hypertension. J Exp Med 208: 535-548, 2011.

35. Tadic M, Cuspidi C, Bombelli M and Grassi G: Right heart remodeling induced by arterial hypertension: Could strain assessment be helpful? J Clin Hypertens (Greenwich) 20: 400-407, 2018.

36. Maron BAandLoscalzo J:Pulmonary hypertension: Pathophysiology and signaling pathways. Handb Exp Pharmacol 218: 31-58, 2013

37. Meloche J, Le Guen M, Potus F, Vinck J, Ranchoux B, Johnson I, Antigny F, Tremblay E, Breuils-Bonnet S, Perros F, et al: miR-223 reverses experimental pulmonary arterial hypertension. Am J Physiol Cell Physiol 309: C363-C372, 2015.

38. McCann GP, Gan CT, Beek AM, Niessen HW, Vonk Noordegraaf A and van Rossum AC: Extent of MRI delayed enhancement of myocardial mass is related to right ventricular dysfunction in pulmonary artery hypertension. AJR Am J Roentgenol 188: 349-355, 2007.

39. Shehata ML, Lossnitzer D, Skrok J, Boyce D, Lechtzin N, Mathai SC, Girgis RE, Osman N, Lima JA, Bluemke DA, et al: Myocardial delayed enhancement in pulmonary hypertension: Pulmonary hemodynamics, right ventricular function, and remodeling. AJR Am J Roentgenol 196: 87-94, 2011.

40. Hessel MH, Steendijk P, den Adel B, Schutte CI and van der Laarse A: Characterization of right ventricular function after monocrotaline-induced pulmonary hypertension in the intact rat. Am J Physiol Heart Circ Physiol 291: H2424-H2430, 2006.

41. Drake JI, Bogaard HJ, Mizuno S, Clifton B, Xie B, Gao Y, Dumur CI, Fawcett P, Voelkel NF and Natarajan R: Molecular signature of a right heart failure program in chronic severe pulmonary hypertension. Am J Respir Cell Mol Biol 45: 1239-1247, 2011.

42. MacLean J and Pasumarthi KB: Signaling mechanisms regulating fibroblast activation, phenoconversion and fibrosis in the heart. Indian J Biochem Biophys 51: 476-482, 2014.

43. Zhang J, Fan G, Zhao H, Wang Z, Li F, Zhang P, Zhang J, Wang $X$ and Wang W: Targeted inhibition of focal adhesion kinase attenuates cardiac fibrosis and preserves heart function in adverse cardiac remodeling. Sci Rep 7: 43146, 2017. 